\title{
Impacto de la inmunoterapia subcutánea con Dermatophagoides farinae y Dermatophagoides pteronyssinus sobre la calidad de vida de pacientes con rinitis y asma alérgica
}

\author{
Juan José Yepes-Núñez ${ }^{1,2,3}$, Carolina Gómez ${ }^{1,3}$, Yeinis Espinoza ${ }^{1,3}$, Ricardo Cardona ${ }^{1,3}$ \\ 1 Grupo de Alergología Clínica y Experimental, Universidad de Antioquia, Medellín, Colombia \\ 2 Grupo Académico de Epidemiología Clínica, Universidad de Antioquia, Medellín, Colombia \\ ${ }^{3}$ Servicio de Alergología Clínica, Universidad de Antioquia, Medellín, Colombia \\ Institución donde se realizó el trabajo: Servicio de Alergología Clínica, Universidad de Antioquia, Medellín, \\ Colombia.
}

Introducción. Como sucede en otras partes del mundo, la prevalencia de asma y rinitis alérgica en Colombia está en aumento. Se ha establecido que la inmunoterapia subcutánea con alérgenos es eficaz a largo plazo en pacientes con rinitis alérgica y asma sensibilizados a Dermophagoides.

Objetivo. Proveer evidencia sobre los cambios relacionados con la calidad de vida inducidos por la inmunoterapia subcutánea en sujetos con alergia respiratoria.

Materiales y métodos. Se seleccionaron 76 sujetos con diagnóstico de alergia respiratoria con sensibilización a Dermatophagoides farinae y Dermatophagoides pteronyssinus. Para la evaluación de la calidad de vida se emplearon los instrumentos Kidscreen-27 y SF-36 (Short form 36). Estos instrumentos se aplicaron en dos ocasiones: durante la primera visita, en la cual se iniciaba la inmunoterapia subcutánea, y un año después de haberse iniciado el tratamiento.

Resultados. Al año de estar recibiendo la inmunoterapia, los 22 sujetos que completaron el estudio presentaron cambios positivos en términos de calidad de vida. En los niños, el principal cambio se presentó en el dominio del 'entorno escolar' mientras que en los adultos fue en el de la 'función física'.

Discusión. Se evaluaron por primera vez en Colombia los beneficios inducidos por la inmunoterapia subcutánea para ácaros de polvo en la calidad de vida de sujetos con rinitis alérgica y asma mediante los cuestionarios Kidscreen-27 y SF-36. Los resultados proveen evidencia de que la inmunoterapia subcutánea influye positivamente en la calidad de vida en sujetos con rinitis asmática y asma sensibilizados a los ácaros de polvo.

Palabras clave: inmunoterapia, calidad de vida, asma, rinitis, Dermatophagoides farinae, Dermatophagoides pteronyssinus.

doi: http://dx.doi.org/10.7705/biomedica.v34i2.1744

The impact of subcutaneous immunotherapy with Dermatophagoides farinaeand Dermatophagoides pteronyssinus on the quality of life of patients with allergic rhinitis and asthma

\begin{abstract}
Introduction: The prevalence of asthma and allergic rhinitis in Colombia is increasing at the same rate as it is in other parts of the world. It has been determined that allergen-specific subcutaneous immunotherapy is effective in subjects with allergic rhinitis and asthma that are sensitized to house dust mites: Dermatophagoides farinae and Dermatophagoides pteronyssinus.

Objective: To provide evidence on changes in the quality of life of subjects induced by allergen-specific subcutaneous immunotherapy with Dermatophagoides farinae and Dermatophagoides pteronyssinus. Materials and methods: We selected 76 subjects with a diagnosis of respiratory allergy with sensitization to Dermatophagoides farinae and Dermatophagoides pteronyssinus. The instruments used for evaluating the quality of life were Kidscreen-27 and SF-36. These instruments were applied twice for each subject: once during the first visit, and during the twelfth visit corresponding to the oneyear follow-up.

Results: Twenty-two subjects completed this study. After one year of treatment with allergenspecific subcutaneous immunotherapy, we found positive changes in terms of the quality of life. In children, the main change was in the School Environment domain while in adults it was in the Physical Function domain.
\end{abstract}

Contribución de los autores:

Todos los autores participaron de igual forma en la atención de las pacientes, la recolección de los datos, la redacción y la corrección del manuscrito. 
Conclusion: We evaluated, for the first time in Colombia, benefits induced by allergen-specific subcutaneous immunotherapy for dust mites in terms of quality of life in subjects with allergic rhinitis and asthma. These results demonstrated that allergen-specific subcutaneous immunotherapy produces a positive influence on subjects sensitized to dust mites that received allergen-specific subcutaneous immunotherapy to Dermatophagoides farinae and Dermatophagoides pteronyssinus after one year.

Key words: Immunotherapy, quality of life, asthma, rhinitis, Dermatophagoides farinae, Dermatophagoides pteronyssinus.

doi: http://dx.doi.org/10.7705/biomedica.v34i2.1744

En las últimas dos décadas se ha presentado un importante aumento en la prevalencia de las enfermedades alérgicas (1). En Estados Unidos y en el este de Europa se ha estimado una prevalencia hasta de $20 \%$ para las enfermedades alérgicas respiratorias (2). En Suramérica, en el estudio ISAAC se estimó una prevalencia de síntomas relacionados con asma en todas las edades superior a $15 \%$, mientras que la de la rinitis alérgica se calculó en 18,5\% (3). En Colombia la prevalencia se ha estimado entre 12 y $32 \%$ para el asma y la rinitis alérgica, respectivamente, variabilidad que se ha relacionado con las diversas herramientas de medición utilizadas (4).

En cuanto al tratamiento, aunque la eliminación de las fuentes que contienen los alérgenos es un componente importante, algunas veces puede ser poco práctico e insuficiente. Por lo tanto, el manejo de las enfermedades alérgicas respiratorias se centra en el uso de fármacos (antihistamínicos, esteroides inhalados o nasales, broncodilatadores) para controlar la inflamación de las vías respiratorias superiores e inferiores. Aunque estos tratamientos han demostrado ser efectivos y seguros, no ofrecen un beneficio duradero una vez suspendidos, y, además, no logran modificar el curso de la enfermedad (5).

La inmunoterapia específica con alérgenos tiene la capacidad de modificar los mecanismos de las enfermedades alérgicas, lo cual se refleja en una mejoría clínica duradera hasta en $70 \%$ de los pacientes tratados, porcentaje que puede variar de acuerdo con la fuente de los alérgenos y el órgano blanco afectado (6). Es así como en el consenso de la Organización Mundial de la Salud de 1998 (7) y en el reporte del 2008 relativo a la guía de rinitis alérgica y su impacto

\footnotetext{
Correspondencia:

Juan José Yepes-Nuñez, McMaster University, HSC-2C1, 1280

Main Street West, Hamilton, ON, L8S 4K1, Canada

Teléfono: 905.525.9140, extensión 22876

yepesnjj@mcmaster.ca
}

Recibido: 20/07/13; aceptado: 10/03/14 sobre el asma (ARIA) (8), quedó establecido que la inmunoterapia específica con alérgenos puede modificar enfermedades alérgicas tales como la rinitis alérgica y el asma producida por pólenes (9). Además, se ha determinado que la inmunoterapia subcutánea específica con alérgenos es eficaz como tratamiento a largo plazo en sujetos con rinitis alérgica y asma sensibilizados a ácaros del género Dermatophagoides. En estos sujetos las principales ventajas de la inmunoterapia subcutánea comprenden la reducción de los síntomas causados por la sensibilización específica durante la exposición al alérgeno y la prevención de sensibilizaciones adicionales o el empeoramiento del asma (10).

Desde el punto de vista de la salud pública, se ha demostrado que las enfermedades alérgicas tienen un efecto perjudicial sobre la calidad de vida, pues alteran el desarrollo personal, la carrera profesional y el estilo de vida tanto de los sujetos como de sus familias (11). Recientemente, los médicos y los investigadores han venido empleando un grupo de instrumentos específicos para evaluar los diferentes dominios de la calidad de vida relacionada con la salud de las personas alérgicas. Las conclusiones en este campo muestran la magnitud del problema y la necesidad urgente de soluciones. Cuando se utiliza la inmunoterapia alergénica adecuadamente, después de un diagnóstico cuidadoso y con extractos bien caracterizados y de buena calidad, este tratamiento puede modificar positivamente la vida de las personas que viven con enfermedades alérgicas (12). El presente estudio provee evidencia sobre los cambios en la calidad de vida inducidos por la inmunoterapia subcutánea con $D$. farinae y $D$. pteronyssinus en personas con alergia respiratoria.

\section{Materiales y métodos}

\section{Diseño del estudio}

Se llevó a cabo un estudio descriptivo prospectivo a partir de una serie de casos de individuos con diagnóstico de alergia respiratoria. La duración del estudio fue de 12 meses por sujeto. El tamaño de la muestra se calculó con el fin de evaluar el 
cambio en la calidad de vida de los sujetos del estudio que recibirían inmunoterapia subcutánea con $D$. farinae y $D$. pteronyssinus durante un año. Dado que no se dispone de estrategias de cálculo del tamaño de la muestra para investigaciones en las que se utilice el instrumento Kidscreen, esta se calculó usando el instrumento SF-36 y con base en los siguientes criterios: error de tipo 1: 0,05; error de tipo 2: 0,20; potencia: $80 \%$. El valor promedio esperado en la primera intervención fue de 50 puntos y el de la segunda intervención de 59 puntos, es decir, una diferencia de nueve puntos, la cual es clínicamente significativa para la intervención. El tamaño total de la muestra se fijó en 147 sujetos (13). En la primera parte de este estudio, publicada recientemente, se describieron las características clínicas y de calidad de vida basales de las personas con alergia respiratoria que hicieron parte de esta investigación (14).

\section{Administración de la inmunoterapia}

Se emplearon extractos estandarizados biológicamente en unidades $\mathrm{DPP} / \mathrm{ml}$ de $D$. farinae y $D$. pteronyssinus (concentración de ácaros de 100 $\mathrm{DPP} / \mathrm{ml}$, Laboratorios Leti, España). La inmunoterapia subcutánea se administró con base en el esquema rápido (rush immunotherapy) (15) con una frecuencia mensual hasta completar 12 dosis, lo que corresponde a un año de seguimiento para cada sujeto. La inmunoterapia subcutánea se administró en el Servicio de Alergología Clínica de la Universidad de Antioquia, con extractos alergénicos suministrados por el sistema de salud colombiano y de acuerdo con las normas internacionales $(9,16)$.

\section{Población de estudio}

Los criterios de inclusión fueron los siguientes: historia clínica sugestiva de alergia respiratoria a ácaros de polvo establecida por alergólogos con base en los criterios de la Global Initiative for Asthma (GINA) y de la iniciativa Allergic Rhinitis and its Impact on Asthma (ARIA) $(8,17)$, así como en la prueba de punción positiva frente a ácaros de polvo usando extractos alergénicos estandarizados para aeroalérgenos comunes de acuerdo con la normativa internacional (18), el pico de flujo nasal inspiratorio y el pico de flujo espiratorio (19), también determinados según los parámetros internacionales estandarizados (20). Los criterios de exclusión fueron aquellos propuestos por la World Allergy Organization (WAO) para la inmunoterapia alergénica (21).
La investigación fue aprobada por el Comité de Ética de la Universidad de Antioquia, y todos los pacientes firmaron un consentimiento informado antes de participar en el estudio.

\section{Pico de flujo nasal inspiratorio}

El pico de flujo nasal inspiratorio es una herramienta sencilla, barata y no invasiva que mide de manera objetiva el flujo nasal (22) con valores expresados en litros por minuto (L/minuto) y se define como el máximo flujo de aire logrado durante una inspiración forzada a través de la nariz (23). Se ha propuesto como normal un valor superior a $150 \mathrm{~L} /$ minuto (24).

\section{Pico de flujo espiratorio}

El pico de flujo espiratorio constituye un elemento útil para la monitorización de la obstrucción de la vía respiratoria cuando no se dispone de espirómetro (25). Los valores pronóstico de normalidad se han determinado de acuerdo con la edad, el sexo y el peso (26).

\section{Evaluación de la calidad de vida}

Para la evaluación de la calidad de vida de los pacientes entre 8 y 18 años de edad, se empleó el instrumento Kidscreen-27 y, para de la de los mayores de 18 años, el SF-36.

El SF-36 es un instrumento genérico que describe la calidad de vida en la población adulta y ha sido validado para la alergia respiratoria (27). Comprende 36 ítems que corresponden a ocho dominios: función física, desempeño físico, dolor corporal, salud general, vitalidad, función social, desem-peño emocional y salud mental (28). Estos ocho campos se clasifican en dos grandes medidas de resumen: la del componente físico y la del componente mental. Las puntuaciones de cada dominio se establecen de acuerdo con las respuestas de cada ítem, con valores de 0 a 100, en donde 0 corresponde a la peor percepción en cuanto al estado de salud y, 100, a la mejor percepción.

En la validación de este cuestionario para Colombia los datos omitidos estuvieron entre 0 y $1,5 \%$, las correlaciones ítem-escala superaron 0,48 y la fiabilidad fue mayor de 0,70 . No se presentaron diferencias en los puntajes de escalas según el tipo de aplicación. La fiabilidad entre observadores fue mayor de 0,80 y el test-retest superó el 0,70 y no mostró diferencias significativas en las dos aplicaciones, concluyéndose que el SF-36 era confiable para evaluar la calidad de vida en salud después de su adaptación lingüística para adultos colombianos (28). 
Por otro lado, la herramienta Kidscreen-27 es un cuestionario corto desarrollado en el marco del Proyecto Kidscreen que la Comisión Europea inició en el 2001 con el objetivo principal de estandarizar un instrumento de evaluación de la calidad de vida en niños y adolescentes de la comunidad europea. El cuestionario comprende cinco dimensiones: bienestar físico, bienestar psicológico, relación entre padres y autonomía, soporte social y entorno escolar (29). La puntuación de los dominios es igual a la del SF-36, es decir, entre 0 y 100

Durante su validación en Colombia, las respuestas al cuestionario mostraron que la calidad de vida en todas las dimensiones fue menor en los niños y los adolescentes enfermos, y aún peor en los hospitalizados y en los niños que en las niñas; entre adolescentes fue similar en ambos sexos. Para darle validez al constructo, las medias de cada uno de los grupos fueron diferentes en todas las dimensiones. La validez interna del instrumento fue mayor de 0,7 en todas las dimensiones. En todos los dominios, el coeficiente de correlación entre clases fue superior a 0,87 y mayor de 0,8 en cuanto a la fiabilidad entre observadores, mostrando propiedades psicométricas de fiabilidad y validez adecuadas (30).

Los cuestionarios Kidscreen-27 y SF-36 fueron contestados por los propios participantes en dos ocasiones: durante la primera visita $\left(T_{0}\right)$, en la cual se iniciaba la inmunoterapia subcutánea, y durante la administración de la dosis número $12\left(\mathrm{~T}_{1}\right)$. Los valores de pico de flujo nasal inspiratorio y pico de flujo espiratorio fueron registrados por personal médico en el mismo momento de la aplicación de los instrumentos. La información se obtuvo en el periodo comprendido entre 2009 y 2012.

\section{Métodos estadísticos}

Los cálculos se hicieron con los software Stata ${ }^{\circledR}$, versión 12,1 (College Station, Texas 77845 USA) y Microsoft Excel $2011^{\circledR}$, versión 14,3,1 (Microsoft Corporation, Redmond, WA 98052, EE.UU). El test de Kolmogorov-Smirnov se empleó para el análisis de normalidad de las variables. La estadística descriptiva se expresó en medias con desviación estándar (DE) y los valores promedio entre $T_{0}$ y $T_{1}$ se compararon con la prueba $t$ de Student para dos muestras dependientes. Se empleó el coeficiente de correlación de Pearson para las correlaciones entre los dominios de calidad de vida relacionada con la salud y pico de flujo espiratorio y pico de flujo nasal inspiratorio. Para los análisis se determinó un nivel de confianza de $95 \%$, un error alfa de $5 \%$ y una significación estadística de $p<0,05$.

Este manuscrito siguió las recomendaciones establecidas por el Global Allergy and Asthma European Network (GALEN) para la realización de documentos sobre calidad de vida y alergia respiratoria (31) y, además, se ejecutó de acuerdo con los requisitos establecidos para la investigación en alergología en artículos recientes (19).

\section{Resultados}

\section{Características de la muestra}

En el momento de la primera visita $\left(T_{0}\right), 65$ sujetos cumplían con los criterios de inclusión y fueron entrevistados; 42 tenían una edad entre 8 y 18 años, y de estos, 25 presentaban rinitis alérgica y 17 , rinitis alérgica con asma. En el momento de la administración de la dosis número $12\left(\mathrm{~T}_{1}\right), 22$ sujetos completaron los cuestionarios; 11 de ellos tenían edades entre los 8 y 18 años y de estos, seis tenían diagnóstico de rinitis alérgica y cinco de rinitis alérgica con asma.

Todos los sujetos estaban sensibilizados a $D$. farinae y $D$. pteronyssinus. Tanto en niños como en adultos con asma hubo un mayor valor promedio de pico de flujo nasal inspiratorio al terminar los 12 meses de inmunoterapia subcutánea, lo cual fue estadísticamente significativo con respecto al pico de flujo nasal inspiratorio basal (cuadro 1).

Durante el periodo de observación del estudio no se reportaron efectos adversos sistémicos producidos por la inmunoterapia subcutánea. Solamente $1 \%$ de los pacientes reportó efectos adversos locales como eritema y prurito en el sitio de aplicación, lo cual no afectó la continuidad del tratamiento.

\section{Calidad de vida en niños y en adultos}

Para evaluar la calidad de vida en los pacientes tratados con inmunoterapia subcutánea (tanto niños como adultos), se compararon los valores promedio de los distintos dominios contemplados en el instrumento utilizado. Al año de estar aplicando la inmunoterapia subcutánea, el valor más bajo de calidad de vida en niños se presentó en el dominio de "bienestar psicológico", mientras que el más alto lo hizo en el de "entorno escolar" (cuadro 2). Por otro lado, en los adultos el valor más alto se presentó en el dominio de "función física" y, el más bajo, en la medida de resumen de "salud mental" (cuadro 2). Hubo diferencias estadísticamente significativas entre los valores 
Cuadro 1. Características clínicas basales $\left(T_{0}\right)$ y después de un año de tratamiento $\left(T_{1}\right)$ con inmunoterapia subcutánea para ácaros de polvo en sujetos con alergia respiratoria

\begin{tabular}{|c|c|c|c|c|c|c|}
\hline & \multicolumn{2}{|c|}{$\mathbf{T}_{0}$} & \multicolumn{2}{|c|}{$\mathrm{T}_{1}$} & \multirow[t]{2}{*}{$p^{\star \star}$} & \multirow[t]{2}{*}{$p^{* * *}$} \\
\hline & Niños & Adultos & Niños & Adultos & & \\
\hline Edad* & $12,21(2,42)$ & $36,22(13,03)$ & $13,55(2,54)$ & $44,64(10,03)$ & $\mathrm{N} / \mathrm{A}$ & $\mathrm{N} / \mathrm{A}$ \\
\hline Hombre & $54,80 \%$ & $26,10 \%$ & $45,50 \%$ & $27,30 \%$ & $\mathrm{~N} / \mathrm{A}$ & $\mathrm{N} / \mathrm{A}$ \\
\hline \multicolumn{7}{|l|}{ Diagnóstico } \\
\hline Rinitis alérgica & $38,46 \%$ & $23,08 \%$ & $27,27 \%$ & $18,18 \%$ & $\mathrm{~N} / \mathrm{A}$ & $\mathrm{N} / \mathrm{A}$ \\
\hline Rinitis alérgica-asma & $26,15 \%$ & $12,31 \%$ & $22,72 \%$ & $31,83 \%$ & $\mathrm{~N} / \mathrm{A}$ & $\mathrm{N} / \mathrm{A}$ \\
\hline \multicolumn{7}{|l|}{ PFE (L/minuto)* } \\
\hline Rinitis alérgica & $324,99(71,10)$ & $352,74(131,10)$ & $305,55(60,35)$ & $348,33(11,78)$ & 0,199 & - \\
\hline Rinitis alérgica-asma & $301,47(83,04)$ & $371,24(171,67)$ & $298,32(72,70)$ & $369,99(185,39)$ & 0,062 & 0,169 \\
\hline \multicolumn{7}{|l|}{ PFNI (L/minuto)* } \\
\hline Rinitis alérgica & $149,04(65,93)$ & $151,94(64,01)$ & $125,83(68,16)$ & $118,33(35,35)$ & 0,121 & 0,906 \\
\hline Rinitis alérgica-asma & $110,83(46,91)$ & $175,41(82,65)$ & $129,16(46,25)$ & $177,61(89,02)$ & 0,043 & 0,020 \\
\hline
\end{tabular}

PFE: pico de flujo espiratorio; PFNI: pico de flujo nasal inspiratorio; N/A: no aplica

*: media y desviación estándar; **: prueba t para diferencia de medias en muestras dependientes entre niños; ${ }^{* * *}$ : prueba t para diferencia de medias en muestras dependientes entre adultos.

Cuadro 2. Valores promedio* de calidad de vida basales $\left(T_{0}\right)$ y después de un año de inmunoterapia subcutánea ( $T 1$ para ácaros de polvo en niños y adultos con alergia respiratoria

\begin{tabular}{|c|c|c|c|}
\hline & \multicolumn{2}{|c|}{ Total } & \multirow[b]{2}{*}{$\mathbf{p}^{* *}$} \\
\hline & $\mathrm{T}_{0}$ & T1 & \\
\hline \multicolumn{4}{|l|}{ Kidscreen-27 } \\
\hline Bienestar físico & $48,59 \quad(5,82)$ & $48,51 \quad(6,74)$ & 0,978 \\
\hline Bienestar psicológico & $40,18 \quad(1,92)$ & $38,64 \quad(1,82)$ & 0,033 \\
\hline Relación con los padres y autonomía & $51,25(11,27)$ & $51,41 \quad(6,75)$ & 0,965 \\
\hline Soporte social y rechazo & $50,60 \quad(8,43)$ & $53,45 \quad(9,93)$ & 0,358 \\
\hline Entorno escolar & $50,27 \quad(8,98)$ & $55,54 \quad(6,68)$ & 0,213 \\
\hline \multicolumn{4}{|l|}{ SF-36 } \\
\hline Función física & $82,27(16,63)$ & $81,36(19,24)$ & 0,742 \\
\hline Desempeño físico & $68,18(44,84)$ & $79,54(36,77)$ & 0,242 \\
\hline Dolor corporal & $72 \quad(28,68)$ & $78,63(23,43)$ & 0,075 \\
\hline Salud general & $59,09(21,68)$ & $66,18(23,92)$ & 0,052 \\
\hline Salud mental & $63,63(20,97)$ & $62,90(21,84)$ & 0,831 \\
\hline Desempeño emocional & $57,57(44,94)$ & $75,75(36,79)$ & 0,111 \\
\hline Función social & $71,59(20,98)$ & $78,40(20,98)$ & 0,140 \\
\hline Vitalidad & $61,81(16,77)$ & $70 \quad(21,67)$ & 0,149 \\
\hline Salud física (PCS) & $49,27 \quad(9,66)$ & $50,88 \quad(8,79)$ & 0,018 \\
\hline Salud mental (MCS) & $40,91(10,27)$ & $44,51 \quad(8,72)$ & 0,113 \\
\hline
\end{tabular}

PCS: medida de resumen del componente físico (Physical Component Summary).

MCS: medida de resumen del componente mental (Mental Component Summary)

*: media y desviación estándar; **: prueba t para diferencia de medias entre muestras dependientes

promedio del dominio de "bienestar psicológico" y la medida de resumen del "componente físico" ( $p=0,03$ y $p=0,01$, respectivamente). Hubo una diferencia mínima clínicamente significativa con la inmunoterapia subcutánea en los dominios incluidos en el SF-36, de 11,36 en los relativos al "desempeño físico" y de 18,18 en los relativos al "desempeño emocional". No se establecieron dichas diferencias mínimas en los sujetos a quienes se les aplicó el Kidscreen-27, debido a que no se encontraron reportes de los valores de dicha diferencia para el instrumento.

\section{Calidad de vida según la enfermedad respiratoria alérgica}

Considerando la enfermedad respiratoria alérgica, en el cuadro 3 se presentan los valores promedio de los dominios de la calidad de vida en niños y adultos. Al cabo de un año de inmunoterapia subcutánea, los pacientes que tenían rinitis alérgica con asma presentaron, en general, mejores puntuaciones que aquellos que solamente tenían rinitis alérgica. No se presentaron cambios estadísticamente significativos entre los valores de los dominios contemplados en el Kidscreen-27 y los incluidos en el SF-36. 
Cuadro 3. Valores promedio* de calidad de vida basales $\left(\mathrm{T}_{0}\right)$ y después de un año de inmunoterapia subcutánea (T1) para ácaros de polvo en niños y adultos con rinitis y asma

\begin{tabular}{|c|c|c|c|c|c|c|}
\hline & & Rinitis & & & itis y asma & \\
\hline & $\mathbf{T}_{0}$ & $\mathbf{T}_{1}$ & $p^{* *}$ & $\mathbf{T}_{0}$ & $\mathbf{T}_{1}$ & $\mathbf{p}^{\star *}$ \\
\hline Kidscreen-27 & & & & & & \\
\hline Bienestar físico & $47,96 \quad(6,79)$ & $49,01 \quad(8,82)$ & 0,649 & $47,15 \quad(6,38)$ & $49,32 \quad(4,05)$ & 0,585 \\
\hline Bienestar psicológico & $39,69 \quad(3,20)$ & $38,53 \quad(2,08)$ & 0,353 & $40,40 \quad(2,22)$ & $39,13 \quad(1,58)$ & 0,060 \\
\hline Relación con los padres y autonomía & $51,35(10,33)$ & $51,93 \quad(8,10)$ & 0,322 & $54,33 \quad(8,30)$ & $52,31 \quad(5,80)$ & 0,365 \\
\hline Soporte social y rechazo & $50,81 \quad(8,91)$ & $47,19 \quad(3,65)$ & 0,486 & $54,45 \quad(8,52)$ & $60,24 \quad(9,59)$ & 0,578 \\
\hline Entorno escolar & $53,37 \quad(9,32)$ & $56,82(10,02)$ & 0,200 & $58,68 \quad(9,86)$ & $57,11 \quad(5,40)$ & 0,835 \\
\hline SF-36 & & & & & & \\
\hline Función física & $78,12(19,39)$ & $77,50(25,33)$ & 0,215 & $87,14(12,19)$ & $83,57(16,76)$ & 0,376 \\
\hline Desempeño físico & $57,81(40,53)$ & $75 \quad(50)$ & 0,391 & $78,57(36,59)$ & $82,14(31,33)$ & 0,356 \\
\hline Dolor corporal & $65,06(25,58)$ & $78,75(27,99)$ & 0,391 & $72,71(28,26)$ & $78,57(22,87)$ & 0,124 \\
\hline Salud general & $55,62(19,37)$ & $57,25(30,56)$ & 0,182 & $60,71(17,23)$ & $71,28(20,08)$ & 0,062 \\
\hline Salud mental & $66,50(21,36)$ & $54 \quad(26,22)$ & 0,650 & $67,42(20,32)$ & $68 \quad(19,18)$ & 0,897 \\
\hline Desempeño emocional & $47,91(45,49)$ & $58,33(50)$ & 0,215 & $71,42(40,49)$ & $85,71(26,22)$ & 0,356 \\
\hline Función social & $69,53(24,98)$ & $71,87(25,76)$ & 0,391 & $76,78(18,29)$ & $82,14(18,89)$ & 0,289 \\
\hline Vitalidad & $55,62(14,70)$ & $70 \quad(14,71)$ & 0,252 & $62,85(20,17)$ & $70 \quad(25,98)$ & 0,381 \\
\hline Salud física (PCS) & $46,52(10,98)$ & $50,97(11,19)$ & 0,492 & $49,99 \quad(8,03)$ & $50,84 \quad(8,13)$ & 0,297 \\
\hline Salud mental (MCS) & $40,43(12,93)$ & $39,22(7,56)$ & 0,189 & $44,01(11,60)$ & $47,54 \quad(8,29)$ & 0,303 \\
\hline
\end{tabular}

PCS: medida de resumen del componente físico (Physical Component Summary).

MCS: medida de resumen del componente mental (Mental Component Summary)

*: media y desviación estándar; **: prueba t para muestras dependientes

En los pacientes con rinitis alérgica, se presentaron diferencias mínimas clínicamente significativas en los siguientes dominios: 17,19 en "desempeño físico", 13,69 en "dolor corporal", 12,5 en "salud mental", 10,42 en "desempeño emocional", y 14,38 en "vitalidad". Con respecto a los pacientes con rinitis alérgica y asma, estas diferencias se presentaron solamente en dos dominios: de 10,57 en "salud general" y de 14,29 en "desempeño emocional".

\section{Correlaciones entre mejoría en la calidad de vida y parámetros clínicos}

Un año después de iniciada la inmunoterapia subcutánea se presentaron correlaciones moderadas y estadísticamente significativas entre los valores promedio del pico de flujo espiratorio y el inspiratorio $(r=0,629, p=0,016)$. Además, los dominios de "componente mental" y de "relación entre padres y autonomía" se relacionaron estrechamente con los valores de pico espiratorio y pico inspiratorio, respectivamente $(r=-0,731, p=0,160$; $r=0,764, p=0,046)$.

En lo tocante al diagnóstico, en los sujetos con rinitis alérgica y asma se presentó una correlación elevada entre los dominios de "salud general" y "salud mental" y los valores del pico inspiratorio $(r=-0,725, p=0,166 ; r=-0,896, p=0,040)$; además, la medida de resumen de "componente físico" y los dominios de "bienestar físico" y "relación entre padres y autonomía", presentaron una estrecha correlación con los valores del pico espiratorio $(r=-0,731, p=0,160 ; r=-0,830, p=0,377 ; r=0,925$, $p=0,247)$ y con los del inspiratorio $(r=-0,796$, $p=0,107 ; r=-0,707, p=0,500 ; r=0,835, p=0,371)$. Solo entre los niños con rinitis alérgica importante se presentaron correlaciones estrechas entre los valores de pico de flujo nasal inspiratorio y los dominios de "relación entre padres y autonomía", "soporte social" y "entorno escolar" $(r=0,862$, $p=0,138 ; r=0,999, p=0,001 ; r=0,844, p=0,156)$.

\section{Discusión}

La administración de un tratamiento, además de cambiar el curso de una enfermedad, como lo hace la inmunoterapia, debe influir positivamente en la percepción que un sujeto tiene acerca de su enfermedad. La inmunoterapia subcutánea con alérgenos es, hasta el día de hoy, el único tratamiento que tiene como objetivo modular la respuesta inmune alérgica $(6,32)$. Por otro lado, el tratamiento con medicamentos antiinflamatorios tiene efectos únicamente sobre los síntomas, sin modificar el curso natural de la enfermedad alérgica.

Hemos evaluado por primera vez en Colombia los beneficios inducidos por la inmunoterapia subcutánea para ácaros de polvo, sobre la calidad de vida de personas con rinitis alérgica y asma por medio de los cuestionarios Kidscreen-27 y SF-36. Los resultados de este estudio proveen evidencia de que la inmunoterapia subcutánea es bien tolerada e influye positivamente en la calidad de vida de personas con rinitis alérgica 
y asma sensibilizadas a los ácaros de polvo, lo cual es importante para justificar un cambio en el tratamiento de los pacientes. Estos resultados coinciden con los de reportes previos sobre los beneficios de la inmunoterapia subcutánea para la calidad de vida de personas aquejadas tanto de rinitis alérgica y como de asma (33-39).

Esta mejoría se midió con los cuestionarios genéricos sobre calidad de vida SF-36 y Kidscreen27. Somos conscientes de que esta es una limitación del estudio, ya que dichos cuestionarios no son específicos para evaluar la calidad de vida relacionada con la salud y ello disminuye su sensibilidad. Sin embargo, estos son los únicos instrumentos que hasta ahora han sido validados para este tipo de estudios en nuestro país.

Por otra parte, el empleo en este estudio del SF-36 permitió comparar el resultado relativo al asma y la rinitis alérgica con otras enfermedades (40). Un beneficio adicional fue conocer la diferencia mínima clínicamente significativa, lo que permitió confirmar que los cambios en los valores promedio entre los dominios del SF-36 superiores a nueve puntos eran clínicamente importantes, justificando, igualmente, subsecuentes modificaciones en el tratamiento de los pacientes (13). En cuanto al Kidscreen-27, hasta la fecha son pocos los estudios que lo usan para evaluar la calidad de vida relacionada con la salud en niños (41), por lo que nuestros resultados pueden aportar al conocimiento de su implementación y comportamiento.

En la literatura médica se ha reiterado la necesidad de hacer estudios sobre los efectos de los tratamientos en la calidad de vida (42). En este sentido, hasta la fecha de aceptación para la publicación del presente manuscrito, tres estudios habían evaluado este resultado de la inmunoterapia subcutánea en sujetos sensibilizados a los ácaros del polvo: dos en personas con asma y uno en personas con rinitis alérgica, pero en ninguno de ellos se aplicó el SF-36 o el Kidscreen-27.

Por medio de un ensayo clínico, Ameal, et al. (36), aplicaron la inmunoterapia subcutánea para $D$. pteronyssinus a 29 sujetos durante 12 meses de seguimiento y encontraron diferencias estadísticamente significativas durante las dos últimas visitas de seguimiento en cuanto a la calidad de vida relacionada con la salud; en el estudio se empleó el cuestionario sobre calidad de vida en el asma (Asthma Quality of Life Questionnaire, AQLA). En un estudio retrospectivo, Gozde Kanmaz, et al., (39) aplicaron la inmunoterapia subcutánea para $D$. pteronyssinus a 52 niños con asma y usaron el cuestionario de calidad de vida para asma en población pediátrica (Pediatric Asthma Quality of Life Questionnaire, PAQLQ). Luego de tres años de seguimiento, se presentó una mejoría en los síntomas relacionados con el asma, en la limitación de actividades y en la puntuación total. Moncayo Coello, et al., (34) evaluaron la calidad de vida relacionada con la salud en niños con rinitis alérgica sensibilizados a epitelio de gato, a Blatella germanica, D. pteronyssinus y Periplaneta americana, quienes recibieron inmunoterapia subcutánea durante un periodo de seguimiento de seis meses. Según los resultados del cuestionario PAQLQ, se presentó una mejoría en la calidad de vida, aunque en el estudio no se aclara en cuáles dominios. Dado que nuestros resultados se obtuvieron aplicando otros instrumentos, no es posible compararlos con los hallazgos reportados en estos estudios (31).

Otras limitaciones de nuestra investigación se refieren al tamaño de la muestra, pues no se captó el número de sujetos requerido y el número de quienes terminaron el estudio fue menor que el de aquellos que lo iniciaron. El número reducido de sujetos que ingresó al estudio se explica por el hecho de que, para la fecha de inicio de la investigación, en la unidad donde se llevó a cabo el proyecto se atendían pocos pacientes que cumplieran con las condiciones necesarias para su inclusión en el programa de inmunoterapia subcutánea. Por otra parte, la pérdida de pacientes en el seguimiento se debió a los cambios en el sitio de atención médica ordenados por el sistema de salud colombiano, hecho que fue ajeno a nuestra voluntad. Debido a los costos que implican los estudios observacionales o experimentales, no fue posible contar con un grupo de control además de los sujetos que recibieron la intervención. Por último, en la mayoría de los casos no fue posible durante el seguimiento obtener información confiable con respecto a otros tratamientos para enfermedades alérgicas respiratorias que los pacientes recibían simultáneamente con la inmunoterapia subcutánea, por lo cual dichos datos no se tuvieron en cuenta en el análisis de los resultados.

Esta investigación es la primera de su tipo en nuestro país. Los hallazgos del presente estudio describen los cambios clínicos positivos en términos de la calidad de vida de personas con alergia respiratoria. Sin embargo, las causas de este impacto son complejas y no pueden adjudicársele solamente a la inmunoterapia subcutánea debido 
a las dificultades metodológicas que acabamos de exponer. Sin embargo, dada la relevancia que puede tener la presente información tanto para el personal de salud como para los pacientes, consideramos importante la publicación de estos hallazgos. Esperamos que los resultados de esta investigación sirvan de base para el desarrollo de otros estudios en este mismo campo, que permitan obtener información adicional acerca del impacto de la inmunoterapia subcutánea en las enfermedades alérgicas respiratorias.

\section{Conflicto de intereses}

Cada uno de los autores declara no tener conflictos de intereses.

\section{Financiación}

El sistema de salud colombiano suministró la inmunoterapia subcutánea.

\section{Referencias}

1. Viswanathan RK, Busse WW. Allergen immunotherapy in allergic respiratory diseases: From mechanisms to metaanalyses. Chest. 2012;141:1303-14. http://dx.doi.org/10. 1378/chest.11-2800

2. Nathan RA. The burden of allergic rhinitis. Allergy Asthma Proc. 2007;28:3-9. http://dx.doi.org/10.2500/aap.2007.28. 2934

3. Chong Neto HJ, Rosário NA, Solé D, Latin American ISAAC Group. Asthma and rhinitis in South America: How different they are from other parts of the world. Allergy Asthma Immunol Res. 2012;4:62-7. http://dx.doi. org/10.4168/aair.2012.4.2.62

4. Dennis RJ, Caraballo L, García E, Rojas MX, Rondón MA, Pérez A, et al. Prevalence of asthma and other allergic conditions in Colombia 2009-2010: A cross-sectional study. BMC Pulm Med. 2012;12:17. http://dx.doi.org/10.1186/1471 2466-12-17

5. Calderón MA, Casale TB, Togias A, Bousquet J, Durham SR, Demoly P. Allergen-specific immunotherapy for respiratory allergies: From meta-analysis to registration and beyond. J Allergy Clin Immunol. 2011;127:30-8. http://dx. doi.org/10.1016/j.jaci.2010.08.024

6. Frati F, Incorvaia C, Lombardi C, Senna G. Allergen immunotherapy: 100 years, but it does not look like. Eur Ann Allergy Clin Immunol. 2012;44:99-106.

7. Bousquet J, Lockey R, Malling HJ. Allergen immunotherapy: Therapeutic vaccines for allergic diseases. A WHO position paper. J Allergy Clin Immunol. 1998;102:558-62. http://dx.doi.org/10.1016/S0091-6749(98)70271-4

8. Bousquet J, Schunemann HJ, Samolinski B, Demoly $P$, Baena-Cagnani CE, Bachert $\mathbf{C}$, et al. Allergic rhinitis and its impact on asthma (ARIA): Achievements in 10 years and future needs. J Allergy Clin Immunol. 2012;130:1049-62. http://dx.doi.org/10.1016/j.jaci.2012.07.053

9. Demoly P, Calderón MA. Dosing and efficacy in specific immunotherapy. Allergy. 2011;66(Suppl.95):38-40. http://dx. doi.org/10.1111/j.1398-9995.2011.02631.x
10. Novak N, Bieber T, Hoffmann M, Folster-Holst R, Homey B, Werfel T, et al. Efficacy and safety of subcutaneous allergen-specific immunotherapy with depigmented polymerized mite extract in atopic dermatitis. J Allergy Clin Immunol. 2012;130:925-31. http://dx.doi.org/10.1016/j.jaci. 2012.08.004

11. Hyland ME. The influence of beliefs on the quality of life of patients with allergic diseases. Clin Exp Allergy. 1999;29:1591-2. http://dx.doi.org/10.1046/j.1365-2222.1999. 00735.x

12. Calderón MA, Demoly P, van Wijk RG, Bousquet J, Sheikh A, Frew A, et al. EAACl: A European declaration on immunotherapy. Designing the future of allergen specific immunotherapy. Clin Transl Allergy. 2012;2:20. http://dx.doi. org/10.1186/2045-7022-2-20

13. Samsa G, Edelman D, Rothman ML, Williams GR, Lipscomb J, Matchar D. Determining clinically important differences in health status measures: A general approach with illustration to the Health Utilities Index Mark II. Pharmacoeconomics. 1999;15:141-55.

14. Yepes-Núñez JJ, Gómez-García C, Espinosa-Herrera Y, Cardona-Villa R. Health-related quality of life in children and adults with respiratory allergy in Colombia: Prospective study. Allergol Immunopathol (Madr). 2012;40:379-84. http:// dx.doi.org/10.1016/j.aller.2011.11.008

15. Brehler R, Klimek L, Pfaar O, Hauswald B, Worm M, Bieber T. Safety of a rush immunotherapy build-up schedule with depigmented polymerized allergen extracts. Allergy Asthma Proc. 2010;31:e31-8. http://dx.doi.org/10.2500/aap. 2010.31.3334

16. Zuberbier T, Bachert C, Bousquet PJ, Passalacqua G, Canonica GW, Merk $\mathrm{H}$, et al. GA(2) LEN/EAACl pocket guide for allergen-specific immunotherapy for allergic rhinitis and asthma. Allergy. 2010;65:1525-30. http://dx.doi. org/10.1111/j.1398-9995.2010.02474.x

17. Kroegel C. Global Initiative for Asthma (GINA) guidelines: 15 years of application. Expert Rev Clin Immunol. 2009;5: 239-49. http://dx.doi.org/10.1586/eci.09.1

18. Bousquet J, Heinzerling L, Bachert C, Papadopoulos NG, Bousquet PJ, Burney PG, et al. Practical guide to skin prick tests in allergy to aeroallergens. Allergy. 2012;67:1824. http://dx.doi.org/10.1111/j.1398-9995.2011.02728.x

19. Papadopoulos NG, Agache I, Bavbek S, Bilo BM, Braido $F$, Cardona V, et al. Research needs in allergy: An EAACl position paper, in collaboration with EFA. Clin Transl Allergy. 2012;2:21. http://dx.doi.org/10.1186/2045-7022-2-21

20. Scadding G, Hellings P, Alobid I, Bachert C, Fokkens W, van Wijk RG, et al. Diagnostic tools in Rhinology EAACl position paper. Clin Transl Allergy. 2011;1:2. http://dx.doi. org/10.1186/2045-7022-1-2

21. Bousquet J, Lockey R, Malling HJ, Álvarez-Cuesta E, Canonica GW, Chapman MD, et al. Allergen immunotherapy: Therapeutic vaccines for allergic diseases. World Health Organization. American Academy of Allergy, Asthma and Immunology. Ann Allergy Asthma Immunol. 1998;81:401-5

22. Nathan RA, Eccles R, Howarth PH, Steinsvag SK, Togias A. Objective monitoring of nasal patency and nasal physiology in rhinitis. J Allergy Clin Immunol. 2005;115(Suppl.1):S44259. http://dx.doi.org/10.1016/j.jaci.2004.12.015 
23. Pertuze J, Watson A, Pride NB. Maximum airflow through the nose in humans. J Appl Physiol.1991;70:1369-76.

24. Hooper RG. Forced inspiratory nasal flow-volume curves: A simple test of nasal airflow. Mayo Clin Proc. 2001;76:990-4. http://dx.doi.org/10.4065/76.10.990

25. Smolensky MH, D’Alonzo GE, Kunkel G, Barnes PJ. Day-night patterns in bronchial patency and dyspnea: Basis for once-daily and unequally divided twice-daily theophylline dosing schedules. Chronobiol Int. 1987;4:303-17.

26. GINA. Global Initiative for Asthma. Fecha de consulta: $1^{\circ}$ de febrero de 2014. Disponible en: http://www.ginasthma.org.

27. Leong KP, Yeak SC, Saurajen AS, Mok PK, Earnest A, Siow JK, et al. Why generic and disease-specific quality-oflife instruments should be used together for the evaluation of patients with persistent allergic rhinitis. Clin Exp Allergy. 2005;35:288-98. http://dx.doi.org/10.1111/j.13652222.2005.02201.x

28. Lugo LH, García HI, Gómez C. Confiabilidad del cuestionario de calidad de vida en salud SF-36 en Medellín, Colombia. Rev Fac Nac Salud Pública. 2006;24:37-50.

29. Ravens-Sieberer U, Auquier P, Erhart M, Gosch A, Rajmil L, Bruil J, et al. The KIDSCREEN-27 quality of life measure for children and adolescents: Psychometric results from a cross-cultural survey in 13 European countries. Qual Life Res. 2007;16:1347-56. http://dx.doi.org/10.1007/s11136007-9240-2

30. Quintero CA, Lugo LH, García HI, Sánchez A. Validación del cuestionario KIDSCREEN-27 de calidad de vida relacionada con la salud en niños y adolescentes de Medellín, Colombia. Rev Colomb Psiquiatr. 2011;40:470-87.

31. Braido F, Bousquet PJ, Brzoza Z, Canonica GW, Compalati E, Fiocchi A, et al. Specific recommendations for PROs and HRQoL assessment in allergic rhinitis and/or asthma: A GA(2)LEN taskforce position paper. Allergy. 2010;65:959-68. http://dx.doi.org/10.1111/j.13989995.2010.02383.x

32. Sánchez J, Restrepo M, Díez S, Cardona R. Comparación del efecto clínico de la inmunoterapia en pacientes con asma alérgica según la edad y el patrón de sensibilización. Alergia, Asma e Inmunologia Pediátricas. 2012;21:77.

33. Álvarez-Cuesta E, Aragoneses-Gilsanz E, MartínGarcía C, Berges-Gimeno P, González-Mancebo E, Cuesta-Herranz J. Immunotherapy with depigmented glutaraldehyde-polymerized extracts: Changes in quality of life. Clin Exp Allergy. 2005;35:572-8. http://dx.doi. org/10.1111/j.1365-2222.2005.02245.x

34. Moncayo CV, Rosas MA, del Río BE, Lerma L, Velázquez Y, Sienra JJ. Quality of life in children with allergic rhinitis before and after being treated with specific immunotherapy (cases and controls). Rev Alerg Mex. 2003;50:170-5.

35. Salazar AA, López AI, Paz D, Galindo JA, Martínez R, Papaqui S. Impact of integrated asthma treatment on the patients' quality of life. Rev Alerg Mex. 2003;50:60-3.

36. Ameal A, Vega-Chicote JM, Fernández S, Miranda A, Carmona MJ, Rondón MC, et al. Double-blind and placebocontrolled study to assess efficacy and safety of a modified allergen extract of Dermatophagoides pteronyssinus in allergic asthma. Allergy. 2005;60:1178-83. http://dx.doi. org/10.1111/j.1398-9995.2005.00862.x

37. Fell WR, Mabry RL, Mabry CS. Quality of life analysis of patients undergoing immunotherapy for allergic rhinitis. Ear Nose Throat J. 1997;76:528-36.

38. Martínez-Olivares R, López-García Al, Paz-Martínez D, Galindo-García JA, Salazar-Aguilar AA, Papaqui-Tapia S. Impact of integral allergic treatment in the quality of life of patients with allergic rhinitis. Rev Alerg Mex. 2002;49:42-5.

39. Gozde H, Harmanci K, Razi C, Kose G, Cengizlier MR. Specific immunotherapy improves asthma related quality of life in childhood. Allergologia et Immunopathologia. 2011;39:68-72. http://dx.doi.org/10.1016/j.aller.2010.04.005

40. Leynaert B, Neukirch C, Liard R, Bousquet J, Neukirch F. Quality of life in allergic rhinitis and asthma. A population-based study of young adults. Am J Respir Crit Care Med. 2000;162:1391-6. http://dx.doi.org/10.1164/ ajrccm.162.4.9912033

41. Ravens-Sieberer U, Schmidt S, Gosch A, Erhart M, Petersen C, Bullinger M. Measuring subjective health in children and adolescents: Results of the European KIDSCREEN/DISABKIDS Project. Psychosoc Med. 2007;4: Doc08.

42. Baiardini I, Braido F, Bindslev-Jensen C, Bousquet PJ, Brzoza Z, Canonica GW, et al. Recommendations for assessing patient-reported outcomes and health-related quality of life in patients with urticaria: A GA(2)LEN taskforce position paper. Allergy. 2011;66:840-4. http://dx.doi.org/10. 1111/j.1398-9995.2011.02580.x 